\title{
A Fast Sparse Reluctance- and Capacitance-Based Solver for the Partial Element Equivalent Circuit Method
}

\author{
Daniele Romano, Giulio Antonini, Senior Member, IEEE, Danesh Daroui, Member, IEEE, \\ and Jonas Ekman, Member, IEEE
}

\begin{abstract}
A new technique for the reluctance method applied to the partial element equivalent circuit method for the time-domain analysis is presented which is well suited to be combined with acceleration techniques. In particular, taking advantage of the rank deficiency of the magnetic and electric field couplings, a new technique for the sparsification of reluctance and capacitance matrices is adopted. Furthermore, the multiscale block decomposition technique has been applied to fast fill these matrices. Finally, the sparse multifrontal LU factorization has been adopted to efficiently compute the global solution. Numerical results demonstrate the validity of the proposed approach in terms of speedup and accuracy.
\end{abstract}

Index Terms-Acceleration techniques, adaptive cross approximation, fast solvers, multiscale block decomposition, partial element equivalent circuit (PEEC), reluctance method, sparse multifrontal LU factorization, time-domain methods.

\section{INTRODUCTION}

$\mathbf{T}$ HREE-DIMENSIONAL electromagnetic models have gained significant importance in virtual prototyping because of their capability to tackle a multitude of signal integrity (SI), power integrity (PI), and electromagnetic compatibility (EMC) problems.

Among the integral equation-based techniques, the method of moments (MoM) and the partial element equivalent circuit (PEEC) method [1] have become popular, the former especially for antenna applications, the latter for mixed electromagnetic circuit problems. Real-life problems are typically characterized by geometrical complexity and high-frequency content of voltage and current signals, resulting in an increasingly high number of unknowns. In this case, many existing full-wave integral equation-based methods for solving large problems adopt iterative solvers [2] since traditional direct methods are computationally expensive because their complexity scales as $O\left(N^{3}\right)$, where $N$ is the number of unknowns. Furthermore, the storage

Manuscript received January 9, 2014; revised February 27, 2014; accepted March 29, 2014. Date of publication May 8, 2014; date of current version September 26, 2014.

D. Romano and G. Antonini are with the UAq EMC Laboratory, Dipartimento di Ingegneria Industriale e dell'Informazione e di Economia, Università degli Studi dell'Aquila, 67100 L'Aquila, Italy (e-mail: giulio.antonini@univaq.it; daniele.romano.vis@gmail.com).

D. Daroui is with SP Technical Research Institute of Sweden, SE-501 15 Borås Sweden (e-mail: danesh.daroui@ combitech.se).

J. Ekman is with the Department of Computer Science, Electrical and Space Engineering, Luleå University of Technology, SE-971 87 Luleå, Sweden (email: jonas.ekman@ltu.se).

Color versions of one or more of the figures in this paper are available online at http://ieeexplore.ieee.org.

Digital Object Identifier 10.1109/TEMC.2014.2314715 matrix fill-in and matrix-vector multiplication operations scale as $O\left(N^{2}\right)$ and, for large $N$, it can be still time-demanding. To overcome such limitations, over the last 20 years, several techniques and algorithms have been proposed to be combined with iterative solvers which have dramatically reduced the computational cost. The list includes the fast multipole method (FMM) in its single level [3]-[5] and multilevel [2], [6]-[8] versions. One major drawback of these approaches is their dependence on the integral equation kernel.

A possible alternative is represented by rank-based techniques that exploits the rank-deficient nature of the coupling matrix blocks representing well-separated interactions. In this class of techniques fall the singular value decomposition (SVD), the QR decomposition methods [9]-[11], and the adaptive cross approximation (ACA) [12], [13]. All these techniques are purely algebraic kernel-function-independent methods which are also easy to be implemented. They have been widely used to accelerate MoM frequency-domain solution, especially for scattering problems involving perfect electric conducting objects.

The PEEC approach has been used in different forms for the quasi-static and full-wave electromagnetic models [1], [14]-[16], and, like other integral equation-based techniques, its complexity scales as $O\left(N^{3}\right)$ and $O\left(N^{2}\right)$ for direct and iterative solvers, respectively. The PEEC method has been accelerated by means of the FMM technique, as described in [17] and [18] for both the frequency and the time domain. The $\mathrm{QR}$ decomposition has also been used to speed up the computation of partial inductances [19]. In [20], a block-partitioned Gauss-Seidel PEEC solver accelerated by QR-based coupling matrix compression techniques is presented for frequency-domain analysis.

Another possible alternative to accelerate the PEEC solver is represented by the sparsification of dense matrices, specifically partial inductance and coefficients of potential matrices. It is known that this easily leads to poor accuracy because both matrices do not exhibit significant locality. More effective is the sparsification of their inverse, named reluctance and capacitance matrices because these matrices are characterized by a much better locality property. This property has been discussed for the reluctance matrix in [21] and [22], and some qualitative explanation has been provided. The main issue with this approach is that the computation of reluctance and capacitance matrices is computationally expensive for large problems and becomes not reasonable for problems exceeding some ten thousands of unknowns since this step scales as $O\left(N^{3}\right)$. Furthermore, the sparsification requires computing some coupling coefficient to 
be compared with a threshold fixed by the user. This step may also result to be computationally expensive for large problems since it scales as $O\left(N^{2}\right)$.

In this paper, a new sparsification strategy of PEEC matrices is presented. Indeed, the multiscale block decomposition method is used to efficiently invert the partial inductances and coefficients of potential matrices. Furthermore, an effective reordering algorithm of basis functions is adopted to better separate strong from weak-coupled basis functions, thus improving the sparsification step. As a result, the reluctance and capacitance matrices can be sparsified without explicitly scanning the coupling coefficients and automatically obtaining significant sparsity.

This paper is organized as follows. The reluctance- and capacitance-based PEEC formulation is reviewed in Section II. Also, the proposed sparsification strategy and the actual state of the art are presented in Section II. The multiscale block decomposition technique that is used to efficiently invert the partial inductance and coefficient of potential matrices is briefly reviewed in Section III along with the ACA compression technique. The numerical results described in Section IV prove the accuracy and efficiency of the proposed technique. The conclusions are drawn in Section V.

\section{Reluctance- AND CAPACITANCE-BASEd PEEC FORMULATION}

\section{A. PEEC Time-Domain Formulation}

The derivation of PEEC equations for a system constituted by conductors and dielectrics can be summarized as follows (the detailed description is given in [24]). Let be:

1) $\mathbf{v}$ the $n \times 1$ vector of node potentials;

2) i the $m \times 1$ vector of currents;

3) $\mathbf{q}_{d}$ the $m_{d} \times 1$ vector of charges;

4) A the $m \times n$ connectivity matrix;

5) $\mathbf{L}_{p}$ the $m \times m$ partial inductance matrix;

6) $\mathbf{P}$ the $n \times n$ coefficient of potential matrix;

7) $\mathbf{G}$ the $n \times n$ lumped element conductance matrix;

8) $\mathbf{R}$ the $m_{c} \times m$ resistance matrix of conductors;

9) $\mathbf{C}_{d}$ the $m_{d} \times m_{d}$ excess capacitance matrix of dielectrics;

10) $\mathbf{i}_{s}$ the $n \times 1$ vector of current sources;

11) $\mathbf{v}_{s}$ the $m \times 1$ vector of voltage sources induced by external fields;

where $n$ is the number of nodes, $m$ is the number of branches, $m_{c}$ is the number of conductor branches and $m_{d}$ is the number of dielectric branches. Let $\left[\mathbf{i}, \mathbf{v}, \mathbf{q}_{d}\right]^{T}$ be the unknown vector and $m_{c}$ and $m_{d}$ be the number of conductor and dielectric branches, respectively. By defining the selection matrices $\boldsymbol{\Psi}_{c}$ and $\boldsymbol{\Psi}_{d}$, of size $m \times m_{c}$ and $m \times m_{d}$, respectively, as

$$
\mathbf{\Psi}_{c}=\left[\begin{array}{c}
\mathbf{I}_{d} \\
\mathbf{0}
\end{array}\right] \quad \mathbf{\Psi}_{d}=\left[\begin{array}{c}
\mathbf{0} \\
\mathbf{I}_{d}
\end{array}\right]
$$

where $I_{d}$ denotes the identity matrix; the generalized Kirchoff's laws, for dielectrics and conductors, can be written as

$$
-\mathbf{A v}(t)-\Psi_{c} \mathbf{R i}(t)-\mathbf{L}_{p} \frac{d \mathbf{i}(t)}{d t}-\boldsymbol{\Psi}_{d} \mathbf{C}_{d}^{-1} \mathbf{q}_{d}(t)=\mathbf{v}_{s}(t)
$$

$$
\begin{aligned}
& \mathbf{C} \frac{d \mathbf{v}(t)}{d t}+\mathbf{G} \mathbf{v}(t)-\mathbf{A}^{T} \mathbf{i}(t)=\mathbf{i}_{s}(t) \\
& \frac{d \mathbf{q}_{d}(t)}{d t}-\mathbf{\Psi}_{d}^{T} \mathbf{i}(t)=\mathbf{0}
\end{aligned}
$$

where $\mathbf{C}=\mathbf{P}^{-1}$.

These equations can be solved by a number of numerical methods which can be explicit or implicit [23], direct or iterative. In this paper, we will focus on the Backward Euler scheme which is implicit, but it is worth to notice that the technique that will be presented can be applied to any implicit scheme. Hence, (2), at time step $k$, can be written as

$$
\begin{aligned}
& -\mathbf{A v}_{k}-\left[\mathbf{\Psi}_{c} \mathbf{R}+\frac{\mathbf{L}_{p}}{h}\right] \mathbf{i}_{k}+-\mathbf{\Psi}_{d} \mathbf{C}_{d}^{-1} \mathbf{q}_{d_{k}}=\mathbf{v}_{s_{k}}-\frac{\mathbf{L}_{p}}{h} \mathbf{i}_{k-1} \\
& {\left[\frac{\mathbf{C}}{h}+\mathbf{G}\right] \mathbf{v}_{k}-\mathbf{A}^{T} \mathbf{i}_{k}=\mathbf{i}_{s_{k}}+\frac{\mathbf{C}}{h} \mathbf{v}_{k-1}} \\
& \frac{\mathbf{I}_{d}}{h} \mathbf{q}_{d_{k}}-\mathbf{\Psi}_{d}^{T} \mathbf{i}_{k}=\frac{\mathbf{q}_{d_{k-1}}}{h}
\end{aligned}
$$

where $h$ is the time-step size. At time step $k$, (3) can be rewritten in a more compact form as

$$
\begin{gathered}
\underbrace{\left[\begin{array}{ccc}
-\mathbf{A} & -\left[\mathbf{\Psi}_{c} \mathbf{R}+\frac{\mathbf{L}_{p}}{h}\right] & -\mathbf{\Psi}_{d} \mathbf{C}_{d}^{-1} \\
{\left[\frac{\mathbf{C}}{h}+\mathbf{G}\right]} & -\mathbf{A}^{T} & \mathbf{0} \\
\mathbf{0} & -\mathbf{\Psi}_{d}^{T} & \frac{\mathbf{I}_{d}}{h}
\end{array}\right]}_{\mathbf{F}} \underbrace{\left[\begin{array}{c}
\mathbf{v}_{k} \\
\mathbf{i}_{k} \\
\mathbf{q}_{d_{k}}
\end{array}\right]}_{\mathbf{X}} \\
=\underbrace{\left[\begin{array}{c}
\mathbf{v}_{s_{k}}-\frac{\mathbf{L}_{p}}{h} \mathbf{i}_{k-1} \\
\mathbf{i}_{s_{k}}+\frac{\mathbf{C}}{h} \mathbf{v}_{k-1} \\
\frac{\mathbf{q}_{d_{k-1}}}{h}
\end{array}\right]}_{\mathbf{B}} .
\end{gathered}
$$

Assuming zero-state vector at time step $k=1$, at each time step $k$, the solution reads

$$
\mathbf{X}=\mathbf{F}^{-1} \mathbf{B}
$$

thus requiring the inversion or the LU decomposition of matrix F. Since we restrict our analysis to linear terminations, the inverse or the LU decomposition of matrix $\mathbf{F}$ can be computed just once at the beginning of the time sweep. It is well known that this operation is time-consuming having a complexity $O\left(N^{3}\right)$, with $N$ the total number of unknowns.

\section{B. PEEC Modeling With Partial Reluctances}

For quasi-static PEEC models, the inverted matrix of $\mathbf{L}_{p}$ for the inductive couplings, namely partial reluctance matrix $\mathbf{K}$, can be used

$$
\mathbf{K}=\mathbf{L}_{p}^{-1} .
$$


By using this approach, the system of equation (4) can be rewritten as follows:

$$
\begin{aligned}
& \underbrace{\left[\begin{array}{ccc}
-\mathbf{K A} & -\left[\mathbf{K} \boldsymbol{\Psi}_{c} \mathbf{R}+\frac{\mathbf{I}_{d}}{h}\right] & -\mathbf{K} \boldsymbol{\Psi}_{d} \mathbf{C}_{d}^{-1} \\
{\left[\frac{\mathbf{C}}{h}+\mathbf{G}\right]} & -\mathbf{A}^{T} & \mathbf{0} \\
\mathbf{0} & -\mathbf{\Psi}_{d}^{T} & \frac{\mathbf{I}_{d}}{h}
\end{array}\right]}_{\mathbf{F}_{k}} \\
& \times \underbrace{\left[\begin{array}{c}
\mathbf{v}_{k} \\
\mathbf{i}_{k} \\
\mathbf{q}_{d_{k}}
\end{array}\right]}_{\mathbf{X}}=\underbrace{\left[\begin{array}{c}
\mathbf{K} \mathbf{v}_{s_{k}}-\frac{\mathbf{I}_{d}}{h} \mathbf{i}_{k-1} \\
\mathbf{i}_{s_{k}}+\frac{\mathbf{C}}{h} \mathbf{v}_{k-1} \\
\frac{\mathbf{q}_{d_{k-1}}}{h}
\end{array}\right]}_{\mathbf{B}_{k}} .
\end{aligned}
$$

The advantage of this formulation is based on the dominance of the diagonal elements of $\mathbf{K}$. Also, the capacitance matrix $\mathbf{C}$ has this property. Hence, the off-diagonal elements in $\mathbf{K}$ and $\mathbf{C}$ matrices decrease faster than that of the partial inductance and coefficient of potential matrices, $\mathbf{L}_{p}$ and $\mathbf{P}$, respectively. Thus, it can be claimed that $\mathbf{K}$ and $\mathbf{C}$ matrices have better locality than their inverse counterparts, $\mathbf{L}_{p}$ and $\mathbf{P}$ matrices. This property makes it possible to sparsify both these matrices without affecting significantly the accuracy. Hence, it is only needed to keep a small number of elements of the starting matrices $\mathbf{K}$ and $\mathbf{C}$ and still maintaining an appropriate level of accuracy after the sparsification, in order to have

$$
\begin{aligned}
\mathbf{K L} \mathbf{L}_{p} & \approx \mathbf{I}_{d} \\
\mathbf{C P} & \approx \mathbf{I}_{d} .
\end{aligned}
$$

The stability of the method is directly related to how the structure is discretized. It has been proven that the stability is assured for a sufficiently discretized structure [21].

\section{Reluctance Computation for Orthogonal Geometries}

The computation of matrix $\mathbf{K}$ can be accelerated in the case of orthogonal geometries which admit Manhattan-type meshes with orthogonal volume and surface discretization. Indeed, in this case, the vector basis functions for the current are mutually orthogonal, leading to a large number of zero coupling.

In order to reduce the complexity of the matrix inversion taking advantage of the zero couplings, a permutation can always be identified and applied to rows and columns of $\mathbf{L}_{p}$ leading to the following interleaved matrix:

$$
\hat{\mathbf{L}}_{p}=\left[\begin{array}{ccc}
\mathbf{L}_{p_{x}} & \mathbf{0} & \mathbf{0} \\
\mathbf{0} & \mathbf{L}_{p_{y}} & \mathbf{0} \\
\mathbf{0} & \mathbf{0} & \mathbf{L}_{p_{z}}
\end{array}\right]
$$

Subblocks $\mathbf{L}_{p_{x}}, \mathbf{L}_{p_{y}}$, and $\mathbf{L}_{p_{z}}$ contain the coefficients of $\mathbf{L}_{p}$ related to $x-, y$-, and $z$-directed basis functions, respectively. Such permutation has the advantage that the inversion of the matrix $\mathbf{L}_{p}$ can be obtained by inverting these three smaller matrices leading to the following reluctance interleaved matrix:

$$
\hat{\mathbf{K}}=\left[\begin{array}{ccc}
\mathbf{L}_{p_{x}}^{-1} & \mathbf{0} & \mathbf{0} \\
\mathbf{0} & \mathbf{L}_{p_{y}}^{-1} & \mathbf{0} \\
\mathbf{0} & \mathbf{0} & \mathbf{L}_{p_{z}}^{-1}
\end{array}\right]
$$

For the sake of simplicity, in the rest of the paper, we will refer to matrices $\mathbf{L}_{p}$ and $\mathbf{K}$ instead of matrices $\hat{\mathbf{L}}_{p}$ and $\hat{\mathbf{K}}$, but the algorithm that will be presented must be applied to submatrices $\mathbf{L}_{p_{x}}, \mathbf{L}_{p_{y}}, \mathbf{L}_{p_{z}}, \mathbf{L}_{p_{x}}^{-1}, \mathbf{L}_{p_{y}}^{-1}$, and $\mathbf{L}_{p_{z}}^{-1}$.

\section{Threshold-Based Sparsification}

In [24]-[26], matrix $\mathbf{K}$ is sparsified by using the following criterion: A matrix element $K_{i j}$ is set to zero if $\frac{K_{i j}}{\sqrt{K_{i i} K_{j j}}} \leq \tau_{t}$, where $\tau_{t}$ is a certain truncation value.

For large problems, where the number of unknowns exceeds few thousands, there are several steps that are very timeconsuming. Let be $N$ the size of matrix $\mathbf{L}_{p}$ :

1) the computational complexity for computing the inverse of $\mathbf{L}_{p}$ grows as $O\left(N^{3}\right)$;

2) the computational complexity for the sparsification (executed as described above) grows as $O\left(N^{2}\right)$;

3) there is not a physical criterion to predict the value of $\tau_{t}$, for different structures and different meshes, that guarantees a certain percentage of sparsification. Hence, if either a trialand-error approach or an adaptive strategy are applied, this step also results to be very time consuming.

The same reasoning is applied to the sparsification of the capacitance matrix $\mathbf{C}$.

\section{E. Reluctance and Capacitance Domain-Based Sparsification}

In this section, we present a new efficient method for the sparsification of reluctance and capacitance matrices, named $R e$ luctance and Capacitance Domain-Based Sparsification (RC$D B S$ ), that allows us to sparsify the $\mathbf{K}$ and $\mathbf{C}$ matrices without explicitly computing them and also to predict the percentage of sparsification. The method will be presented for $\mathbf{K}$, but it is similar for $\mathbf{C}$.

The basic idea of this method is based on the fact that the positions of the elements with smaller value in $\mathbf{L}_{p}$ are preserved in matrix $\mathbf{K}$ [27]. Hence, once found the position of these elements in $\mathbf{L}_{p}$, it is sufficient to set to zero the elements of $\mathbf{K}$ in such positions.

The target of the RC-DBS algorithm is to find the position of the elements with smaller value in $\mathbf{L}_{p}$ without scanning such matrix. The first step to achieve this goal is to perform a process of decomposition of the volume containing the system under analysis into a certain number of levels $L$. This decomposition is performed as follows.

1) The Cartesian axis along which the domain (or subdomain) is largest is identified.

2) The domain (or subdomain) is cut in correspondence of the average value of the coordinates of the basis functions for the current on the chosen Cartesian axis.

3) Steps 1 and 2 are repeated recursively for each subdomain, until reaching the final level $L$ of the decomposition. 


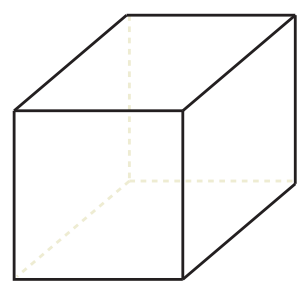

(a)

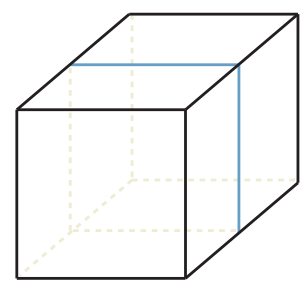

(b)

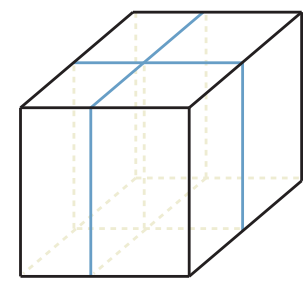

(c)

Fig. 1. Multilevel subdivision of the box enclosing the object. (a) Box enclosing the system. (b) One level of decomposition. (c) Two levels of decomposition.

Differently from the standard oct-tree decomposition, the previous algorithm may lead to subdomains of different size, as shown in Fig. 1.

In this way, assuming an almost uniform mesh, the elements with higher value in $\mathbf{L}_{p}$ are those that represent the interactions between the basis functions inside the same subdomain (because they are physically close), while the elements that represent the interactions between the basis functions belonging to different subdomains exhibit a low-rank behavior, and hence, they represent the smaller values in $\mathbf{L}_{p}$. Thus, once the domain decomposition is performed, it is sufficient to set to zero the values in $\mathbf{K}$ that represent the interactions between the basis functions belonging to different subdomains at the final level $L$.

Let $N$ be the number of basis functions for the current, and, for simplicity, let us assume to have the same number of basis functions inside each box. Then, the percentage of sparsification can be controlled by choosing the number of levels $L$ of decomposition.

1) With one level of decomposition, the sparsification is about $50 \%$.

2) With two levels of decomposition, the sparsification is about $75 \%$.

3) With three levels of decomposition, the sparsification is about $87.5 \%$, and so on.

In order to better understand this statement, Fig. 2 shows the structure of a sparsified matrix $\mathbf{K}$ for three different levels of decomposition. For simplicity, the coefficients that represent the interactions between the basis functions inside the same box have been disposed on diagonal submatrices (the blue subblocks in the figure). Thus, these are the only nonzero elements in $\mathbf{K}$.

The computational complexity of the domain decomposition is $O(L \cdot N)$. For a small number of levels $L$ (with six levels of decomposition the sparsification is about $98 \%$ ), it grows as $O(N)$.

\section{Multiscale Compressed Block Decomposition}

The multiscale compressed block decomposition (MS-CBD) algorithm has been proposed in [28] to accelerate the solution of linear systems in the framework of surface integral equations for the analysis of scattering problems by using the MoM approach.

In [29], a different MS-CBD algorithm has been proposed for volume integral equations solved by the PEEC method. In that work, the MS-CBD algorithm is used to compute the inverse of matrices $\mathbf{L}_{p}$ and $\mathbf{P}$ required to fill the matrix $\mathbf{F}$ [see (4)] or matrix $\mathbf{F}_{k}$ [see (7)].
The target is to improve the computational and storage complexity of the rigorous matrix inversion process by exploiting symmetry, recursion, and matrix compression. This statements will be clarified in the next sections.

The steps required by the MS-CBD algorithm can be summarized as follows:

1) domain partitioning;

2) block partitioned inverse (BPI) algorithm (see Section III-A);

3) matrix compression (see Section III-B).

The initial step of the algorithm is to perform the domain decomposition reported in Section II-E with the only difference that the number of levels $L$, as suggested in [28], is computed as

$$
L=\operatorname{ceil}\left(\log _{2}(N / 600)\right)
$$

where $\operatorname{ceil}(x)$ denotes the first integer larger than $x$ and $N$ is the size of the matrix to invert.

\section{A. BPI Algorithm}

The computation of the inverse of matrix $\mathbf{L}_{p}$ or matrix $\mathbf{P}$ (that are symmetric) can be performed by using the BPI algorithm [30]. Let us consider the matrix $\mathbf{L}_{p}$ (for $\mathbf{P}$ is similar) and let us assume to partition it as

$$
\mathbf{L}_{p}=\left[\begin{array}{ll}
\mathbf{L}_{p_{11}} & \mathbf{L}_{p_{12}} \\
\mathbf{L}_{p_{12}}^{T} & \mathbf{L}_{p_{22}}
\end{array}\right] .
$$

The partitioned inverse matrix of $\mathbf{L}_{p}$ can be written as

$$
\mathbf{K}=\mathbf{L}_{p}^{-1}=\left[\begin{array}{cc}
\widetilde{\mathbf{K}}_{11} & \widetilde{\mathbf{K}}_{12} \\
\widetilde{\mathbf{K}}_{12}^{T} & \widetilde{\mathbf{K}}_{22}
\end{array}\right]
$$

where

$$
\begin{aligned}
\widetilde{\mathbf{K}}_{11} & =\mathbf{L}_{p_{11}}^{-1}+\mathbf{L}_{p_{11}}^{-1} \mathbf{L}_{p_{12}} \widetilde{\mathbf{K}}_{22} \mathbf{L}_{p_{12}}^{T} \mathbf{L}_{p_{11}}^{-1} \\
\widetilde{\mathbf{K}}_{12} & =-\mathbf{L}_{p_{11}}^{-1} \mathbf{L}_{p_{12}} \widetilde{\mathbf{K}}_{22} \\
\widetilde{\mathbf{K}}_{22} & =\mathbf{W}^{-1}
\end{aligned}
$$

with

$$
\mathbf{W}=\mathbf{L}_{p_{22}}-\mathbf{L}_{p_{12}}^{T} \mathbf{L}_{p_{11}}^{-1} \mathbf{L}_{p_{12}} .
$$

This inversion formula can be applied recursively for inverting submatrices $\mathbf{L}_{p_{11}}$ and $\mathbf{W}$, where the number of iterations $L$ is chosen as reported in (11).

For each level of recursion, the basis functions are reordered according the domain decomposition, in order to dispose the 


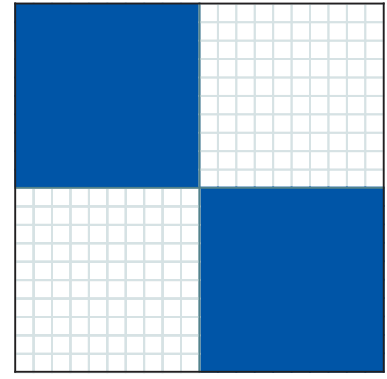

(a)

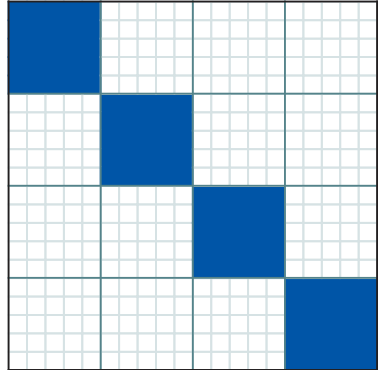

(b)

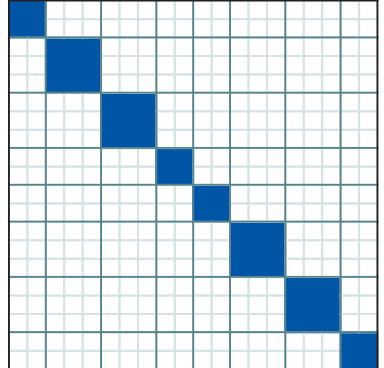

(c)

Fig. 2. Matrix sparsification for three different levels of the decomposition. (a) One level of decomposition. (b) Two levels of decomposition. (c) Three levels of decomposition.

self-interactions (interactions between the basis functions inside the same subdomain) on diagonal subblocks and to dispose the interactions between the basis functions belonging to different subdomains on off-diagonal subblocks. In this way, the offdiagonal subblocks will exhibit a low-rank behavior, and hence, they can be efficiently compressed by using the strategy reported in the next section.

\section{B. Compression Strategy}

Partial inductance and coefficients of potential matrices, $\mathbf{L}_{p}$ and $\mathbf{P}$, respectively, exhibit a low-rank behavior because of the 3-D free space Green's function [19], [20] underlying their evaluation. As a consequence, the subblocks of these matrices that represent the far-field interactions can be efficiently compressed preserving the accuracy.

Let us consider an off-diagonal subblock $\mathbf{M}_{m \times n}$ of matrix $\mathbf{L}_{p}$ or $\mathbf{P}$ that must be compressed (such subblock contains the farfield interactions for the reasons given in previous subsection). Hence, the problem is to find an approximant $\widetilde{\mathbf{M}}$ of $\mathbf{M}$ such that

$$
\|\mathbf{M}-\widetilde{\mathbf{M}}\| \leq \tau_{c}\|\mathbf{M}\|
$$

where $\tau_{c}$ is a thereshold and $\|\mathbf{M}\|$ denotes the Frobenius norm of $\mathrm{M}$. The solution to this problem can be obtained by using the truncated SVD of M but, since the SVD requires the computation of the entire subblock $\mathrm{M}$ and since its computational complexity grows as $O\left(m^{2} n+n^{3}\right)$, the truncated SVD applied to the entire subblock $\mathbf{M}$ is not the best practical solution.

Another popular compression technique to achieve a matrix low-rank approximation is the ACA [12]. The ACA algorithm allows us to compute a low-rank decomposition of a subblock $\mathbf{M}$ with a finite number of rank-1 approximations leading to a low-rank representation:

$$
\mathbf{M} \cong \widetilde{M}=\mathbf{U V}
$$

with $\mathbf{U} \in \mathcal{C}^{m \times q}$ and $\mathbf{V} \in \mathcal{C}^{q \times n}$, where $q \ll m, n$.

The construction of matrices $\mathbf{U}$ and $\mathbf{V}$ is performed by properly choosing rows and columns of $\mathbf{M}$ until the condition in (16) is satisfied. The advantages given by the ACA algorithm are the following.

1) The subblock $\mathbf{M}$ is not entirely computed, but only some rows and columns of it are needed [12].
2) The computational complexity grows as $O(q(m+n))$ [12].

Finally, another advantage given by the matrix compression is that the matrix products between compressed and uncompressed matrices are accelerated for the following reason. Let us assume that the computational complexity for multiplying two generic matrices $\mathbf{D}_{m \times k} \cdot \mathbf{E}_{k \times n}$ is $O(m k n)$. Hence, the computational complexity for computing the matrix product $\mathbf{H}_{m \times m} \cdot \mathbf{S}_{m \times m}$ is $O\left(m^{3}\right)$. Let us assume to compress matrix $\mathbf{S}$. Then, the previous product reads $\mathbf{H}_{m \times m} \cdot \mathbf{S}_{U m \times q} \cdot \mathbf{S}_{V q \times m}$. By first performing first the product $\mathbf{H} \cdot \mathbf{S}_{U}$ and by multiplying the result by $\mathbf{S}_{V}$, the computational complexity is $O\left(2 m^{2} q\right)$. Therefore, if $q<\frac{m}{2}$, the products between compressed and uncompressed matrices are less expensive than the products between uncompressed matrices. Moreover, by using ACA algorithm, the complexity for the storage of matrix $\mathbf{S}$ decreases from $O\left(m^{2}\right)$ to $O(2 m q)$.

\section{Advantages Given by the MS-CBD Algorithm}

In this section, a brief description of the MS-CBD algorithm has been given. The detailed description and the detailed pseudocode are given in [29]. In [29], experimental results shown that the MS-CBD algorithm further improves the computational complexity and the storage complexity of the traditional techniques. The main features that allows us to obtain these improvements can be summarized as follows.

1) The off-diagonal subblocks of matrices $\mathbf{L}_{p}$ and $\mathbf{P}$ are filled directly in compressed form, and hence, a reduced number of the coefficients of these matrices are computed and allocated (this means a substantial improvement on the filling time and memory requirements).

2) All the algebraic operations required to invert $\mathbf{L}_{p}$ and $\mathbf{P}$ are executed block-by-block in order to improve the computational complexity of all matrix products for the reasons given in Section III-B.

3) By applying the recursive BPI algorithm, the rigorous method of inversion, that is computationally very expensive, is required to invert only the subblocks at the final level of recursion. Since they are usually of small size at the final level, their inversion can be efficiently performed. Furthermore, by partitioning the matrices into subblocks, it is possible exploit the symmetry as much possible, and 


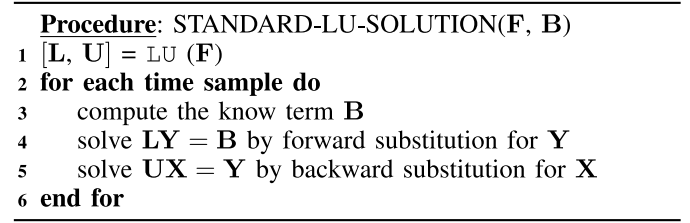

Fig. 3. Pseudocode of procedure STANDARD-LU-SOLUTION.

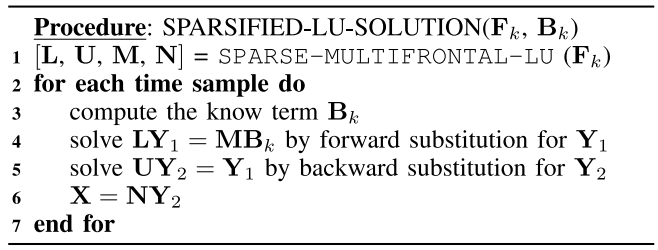

Fig. 4. Pseudocode of procedure SPARSIFIED-LU-SOLUTION.

hence, the allocation of several off-diagonal subblocks can be avoided.

\section{NUMERICAL EXPERIMENTS}

In this section, we evaluate the performance of the reluctance and capacitance method by using different matrix inversion and sparsification schemes. In particular, two different versions of PEEC time-domain solver have been implemented.

1) The first one, denoted as RMI-TBS, that uses the rigorous matrix inversion method to invert $\mathbf{L}_{p}$ and $\mathbf{P}$ and that uses the strategy reported in Section II-D for the sparsification of $\mathbf{K}$ and $\mathbf{C}$. The global solution is computed by using the procedure SPARSIFIED-LU-SOLUTION reported in Section IV-A.

2) The second one, denoted as MS-DBS, that uses the multiscale algorithm to invert $\mathbf{L}_{p}$ and $\mathbf{P}$ and that uses the RC-DBS algorithm (reported in Section II-E) for the sparsification of $\mathbf{K}$ and $\mathbf{C}$. In this case also the global solution is computed by using the procedure SPARSIFIED-LUSOLUTION reported in Section IV-A.

In order to evaluate the accuracy and the performances of RMI-TBS and MS-DBS also the standard time-domain PEEC solver, denoted as MNA, has been implemented. The global solution for MNA is computed by using the procedure STANDARD-LU-SOLUTION reported in Section IV-A. All the experiments have been executed on a PC equipped with 48 GB of RAM and two Intel Xeon quad-core processor operating at $3.46 \mathrm{GHz}$. The code has been developed in MATLAB and all the computations have been performed in double precision.

\section{A. PEEC Solution Computation}

In our experiments, for the standard time-domain PEEC formulation, we have computed the solution for all the time samples of the system shown in (4), by using procedure STANDARDLU-SOLUTION reported in Fig. 3. The input matrix $\mathbf{F}$ and the vector $\mathbf{B}$ represent the matrices of the system (4).

At line 1 of the procedure in Fig. 4, the standard LU decomposition of $\mathbf{F}$ (whose coefficients does not depend on time) is computed, and then, for all the time samples, the solution is computed by exploiting the LU decomposition as reported at lines 2-6.

By applying the reluctance and the capacitance method, assuming that $\mathbf{K}$ and $\mathbf{C}$ are very sparse matrices, the system of equation (7) will be sparse. In order to exploit this sparsification, the solution of (7) has been computed by using the procedure SPARSIFIED-LU-SOLUTION shown in Fig. 4. The input matrix $\mathbf{F}_{k}$ and the vector $\mathbf{B}_{k}$ represent the matrices of the system (7).

At line 1 of the procedure in Fig. 4, the sparse multifrontal LU factorization [31] of $\mathbf{F}_{k}$ (whose coefficients does not depend on time) is computed. In this procedure, the solver will strive to process several independent subsets of the original matrix, where each subset is referred as front. Operations on each front includes dense matrix algorithms which will be efficient in sense of resource utilization. This class of solvers will never create the factors explicitly, but only the parts that are needed will be assembled. Furthermore, the routine presented in Fig. 4 returns the following matrices:

1) the unit lower triangular matrix $\mathbf{L}$;

2) the upper triangular matrix $\mathbf{U}$;

3) a very sparse square matrix $M$ of row permutation;

4) a very sparse square matrix $\mathbf{N}$ of column reordering;

in order to have

$$
\mathbf{M F}_{k} \mathbf{N}=\mathbf{L U} .
$$

The column ordering $\mathbf{N}$ is selected to give a good a priori upper bound on fill-in and then refined during numerical factorization (while preserving the upper bound on fill-in). The row ordering $\mathbf{M}$ is selected during numerical factorization to maintain numerical stability and to preserve sparsity. The process of calculating the permutation matrices is called symbolic factorization, which is a vital process in sparse direct solvers, to reduce the fill-in when factors of the coefficient matrix is being computed. Permutation matrices are used to perform matrix column and row reordering. Different reordering techniques have been introduced such as Approximate Minimum Degree, Cuthil McKee, and Zero-Free Diagonal method, which is recommended for very unsymmetric matrices, to reduce fillin and arithmetic cost [32], [33]. A more detailed discussion of this technique is given in [31]. Finally, at lines 2-7 of the procedure SPARSIFIED-LU-SOLUTION, the solution for all the time samples has been computed by solving the following system:

$$
\mathbf{M}^{-1} \mathbf{L} \mathbf{U N}{ }^{-1} \mathbf{X}=\mathbf{B}_{k} .
$$

Equation (19) has been written by performing the following algebraic operations. From (18), it follows that

$$
\mathbf{F}_{k}=\mathbf{M}^{-1} \mathbf{L U N}^{-1}
$$

and hence, the system (7) can be written as reported in (19). Finally, the solution of (19) is given by

$$
\mathbf{X}=\left(\mathbf{M}^{-1} \mathbf{L} \mathbf{U} \mathbf{N}^{-1}\right)^{-1} \mathbf{B}_{k}
$$

that can be rewritten in equivalent form as follows:

$$
\mathbf{X}=\mathbf{N U}^{-1} \mathbf{L}^{-1} \mathbf{M B}_{k} \text {. }
$$


TABLE I

SCALED UNITS

\begin{tabular}{|c|c|c|c|c|c|c|c|}
\hline Voltage & Current & Charge & $\mathbf{P}$ & $\mathbf{C}_{d}$ & $\mathbf{R}$ & $\mathbf{L}_{p}$ & $\mathrm{~s}$ \\
\hline \hline $\mathrm{V}$ & $\mathrm{mA}$ & $\mathrm{pC}$ & $\mathrm{pF}^{-1}$ & $\mathrm{pF}$ & $\mathrm{k} \Omega$ & $\mu \mathrm{H}$ & $\mathrm{ns}$ \\
\hline
\end{tabular}

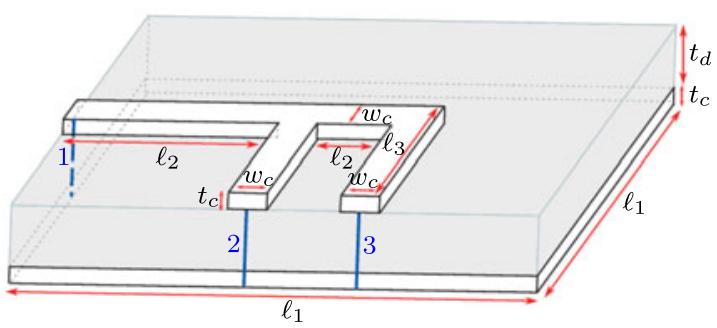

Fig. 5. Structure of the three-port microstrip power-divider circuit.

Finally, the system of equations (7) is typically an illconditioned problem due to the sparsification and due to the fact that the charges are usually much smaller than currents and voltages. In our experiments, we have noted that the conditioning of (7) can be efficiently improved by scaling the units of the electrical quantities as shown in Table I.

\section{B. Three-Port Microstrip Power Divider}

As first example, a three-port microstrip power-divider circuit [34], [35] has been modeled. The conductivity of the microstrip and the ground plane is $\sigma=5.8 \times 10^{7} \mathrm{~S} / \mathrm{m}$. The structure is shown in Fig. 5. The blue lines represent the port. The geometric parameters in Fig. 5 are the following: $\ell_{1}=2 \mathrm{~cm}, \ell_{2}=7.2 \mathrm{~mm}$, $\ell_{3}=1.1 \mathrm{~cm}, w_{c}=0.8 \mathrm{~mm}, t_{d}=0.5 \mathrm{~mm}$, and $t_{c}=50 \mu \mathrm{m}$. The relative permittivity of the dielectric is $\varepsilon_{r}=2.2$. Port 1 is terminated on $10-\Omega$ resistance while ports 2 and 3 are terminated on $64-\Omega$ resistances. A voltage source (amplitude $1 \mathrm{~V}$, rise-time $1 \mathrm{~ns}$, pulsewidth $4 \mathrm{~ns}$ ) drives the first port. Table II shows the performances of MNA, RMI-TBS, and MS-DBS for a different number of unknowns. For the MS-DBS algorithm, the level for sparsification of both the $\mathbf{K}$ and $\mathbf{C}$ matrices has been set equal to 6 and the ACA threshold is set to $10^{-4}$. The third-last row of Table II shows the time spent for the LU factorization of matrix $\mathbf{F}$ [defined in (4)] for the algorithm MNA and of matrix $\mathbf{F}_{k}$ [defined in (7)] for the algorithms RMI-TBS and MSDBS. The second-last row of Table II shows the time spent for the computation of the solution for all the time samples (steps of lines $2-6$ of the procedure STANDARD-LU-SOLUTION, shown in Fig. 3, for the algorithm MNA and steps of lines 2-7 of the procedure SPARSIFIED-LU-SOLUTION, shown in Fig. 4, for the algorithms RMI-TBS and MS-DBS). In order to highlight the difference between the three algorithms, in the last row of Table II, the total time spent to perform the operations reported in all the other rows is reported. Port voltage $V_{p 3}$ for the first case reported in Table II is plotted in Fig. 6. As clearly seen, a very good accuracy is achieved.

\section{Coplanar Striplines}

In the second example, two coplanar striplines are embedded in a dielectric, as shown in Fig. 7 and backed by two metallic

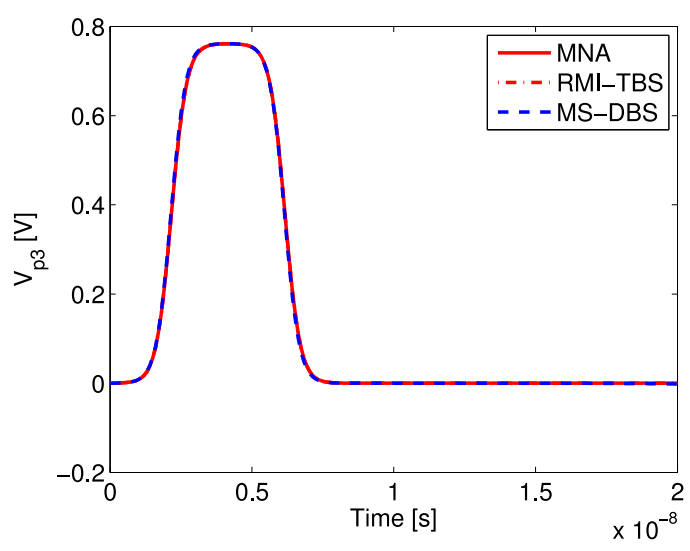

Fig. 6. Port voltages 3 for the three-port microstrip power-divider example.

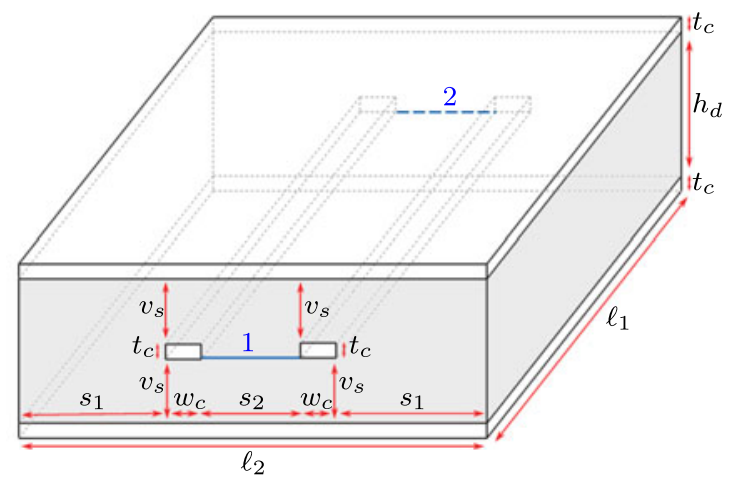

Fig. 7. Structure of the coplanar striplines circuit.

planes. The conductivity of striplines and planes is $\sigma=5.7 \times$ $10^{7} \mathrm{~S} / \mathrm{m}$. The relative permittivity of the dielectric is $\varepsilon_{r}=4$. The blue lines represent the ports. The geometric parameters in Fig. 7 are the following: $\ell_{1}=40 \mathrm{~mm}, \ell_{2}=14 \mathrm{~mm}, s_{1}=$ $5 \mathrm{~mm}, s_{2}=2 \mathrm{~mm}, v_{s}=10 \mathrm{~mm}, h_{d}=20.95 \mathrm{~mm}$, and $t_{c}=$ $50 \mu \mathrm{m}$. All the ports are terminated on $50-\Omega$ resistances. A pseudorandom bit sequence of amplitude $3.3 \mathrm{~V}$ is drawn at port 1 . The simultaneous switching noise is observed at port 2 . Table III shows the performances of MNA, RMI-TBS, and MSDBS for a different number of unknowns. As in the previous example, for MS-DBS algorithm, the level for sparsification of both the $\mathbf{K}$ and $\mathbf{C}$ matrices has been set equal to 6 and the ACA threshold is set to $10^{-4}$. The description of last three rows of Table III is analogous to the description made for Table II. Port voltage $V_{p 2}$ for the first case reported in Table III is plotted in Fig. 8. As clearly seen, a very good accuracy is achieved.

\section{Discussion of the Results}

Tables II and III show that the MS-DBS algorithm always outperforms the MNA and RMI-TBS algorithms. In particular, it is to be pointed out that the MS-DBS algorithm exhibits the better performances than the RMI-TBS algorithm, in all the steps (the multifrontal LU decomposition and solution computation) described in the procedure SPARSIFIED-LUSOLUTION (shown in Fig. 4), in spite of the same percentage of sparsification of $\mathbf{K}$ and $\mathbf{C}$ matrices. This happens because 
TABLE II

EXPERIMENTAL RESUlTS FOR THE THREE-PORT MICROSTRIP POWER-DIVIDER EXAMPLE

\begin{tabular}{|c|c|c|c|c|c|c|c|c|c|}
\hline Number of time samples & \multicolumn{3}{|c|}{200} & \multicolumn{3}{|c|}{200} & \multicolumn{3}{|c|}{200} \\
\hline Size of matrix $\mathbf{L}_{p}$ & \multicolumn{3}{|c|}{11272} & \multicolumn{3}{|c|}{21708} & \multicolumn{3}{|c|}{28112} \\
\hline Size of matrix $\mathbf{P}$ & \multicolumn{3}{|c|}{4502} & \multicolumn{3}{|c|}{8052} & \multicolumn{3}{|c|}{10120} \\
\hline Size of matrix $\mathbf{C}_{d}$ & \multicolumn{3}{|c|}{5400} & \multicolumn{3}{|c|}{10522} & \multicolumn{3}{|c|}{13450} \\
\hline & MNA & RMI-TBS & MS-DBS & MNA & RMI-TBS & MS-DBS & MNA & RMI-TBS & MS-DBS \\
\hline $\mathbf{L}_{p}$ filling time $[\mathrm{s}]$ & 2786 & 2786 & 1259 & 10447 & 10447 & 4255 & 17672 & 17672 & 6918 \\
\hline $\mathbf{L}_{p}$ inversion time $[\mathrm{s}]$ & - & 27 & 8 & - & 216 & 30 & - & 465 & 52 \\
\hline K sparsification time [s] & - & 40 & 1 & - & 145 & 1.7 & - & 247 & 2.2 \\
\hline$\tau_{t}$ for $\mathbf{K}$ sparsification & - & $2.55 \cdot 10^{-4}$ & - & - & $9.5 \cdot 10^{-5}$ & - & - & $6 \cdot 10^{-5}$ & - \\
\hline Sparsity of $\mathbf{K}[\%]$ & - & 98.44 & 98.41 & - & 98.43 & 98.43 & - & 98.35 & 98.41 \\
\hline Memory occupancy $\mathbf{K}$ (or $\mathbf{L}_{p}$ for MNA) [MB] & 683 & 11 & 11 & 2547 & 40 & 40 & 4279 & 71 & 68 \\
\hline $\mathbf{P}$ filling time $[\mathrm{s}]$ & 57 & 57 & 30 & 171 & 171 & 79 & 265 & 265 & 118 \\
\hline $\mathbf{P}$ inversion time $[\mathrm{s}]$ & 14 & 14 & 4 & 83 & 83 & 11 & 159 & 159 & 17 \\
\hline C sparsification time $[\mathrm{s}]$ & - & 12 & 0.54 & - & 38 & 0.76 & - & 60 & 0.9 \\
\hline$\tau_{t}$ for $\mathbf{C}$ sparsification & - & $2.45 \cdot 10^{-4}$ & - & - & $9 \cdot 10^{-5}$ & - & - & $6 \cdot 10^{-5}$ & - \\
\hline Sparsity of $\mathbf{C}[\%]$ & - & 98.42 & 98.39 & - & 98.42 & 98.43 & - & 98.46 & 98.42 \\
\hline Memory occupancy $\mathbf{C}[\mathrm{MB}]$ & 154 & 4.97 & 5.05 & 494 & 15 & 15 & 781 & 24.13 & 24.88 \\
\hline 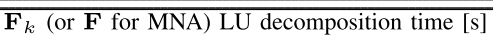 & 56 & 9.15 & 0.65 & 414 & 61.4 & 2.3 & 4374 & 216 & 4.81 \\
\hline Solution time* $[\mathrm{s}]$ & 482 & 19.42 & 2.00 & 1717 & 107 & 6.70 & 2841 & 273 & 12.68 \\
\hline Overall time $[\mathrm{s}]$ & 3395 & 2965 & 1306 & 12832 & 11269 & 4385 & 25311 & 19357 & 7127 \\
\hline
\end{tabular}

TABLE III

EXPERIMENTAL RESULTS FOR THE COPLANAR STRIPLINES EXAMPLE

\begin{tabular}{|c|c|c|c|c|c|c|c|c|c|}
\hline Number of time samples & \multicolumn{3}{|c|}{1569} & \multicolumn{3}{|c|}{1569} & \multicolumn{3}{|c|}{1569} \\
\hline Size of matrix $\mathbf{L}_{p}$ & \multicolumn{3}{|c|}{13840} & \multicolumn{3}{|c|}{19785} & \multicolumn{3}{|c|}{25440} \\
\hline Size of matrix $\mathbf{P}$ & \multicolumn{3}{|c|}{4320} & \multicolumn{3}{|c|}{5644} & \multicolumn{3}{|c|}{6900} \\
\hline Size of matrix $\mathbf{C}_{d}$ & \multicolumn{3}{|c|}{7672} & \multicolumn{3}{|c|}{11127} & \multicolumn{3}{|c|}{14408} \\
\hline & MNA & RMI-TBS & MS-DBS & MNA & RMI-TBS & MS-DBS & MNA & RMI-TBS & MS-DBS \\
\hline $\mathbf{L}_{p}$ filling time [s] & 4458 & 4458 & 1814 & 8729 & 8729 & 3543 & 14513 & 14513 & 5756 \\
\hline $\mathbf{L}_{p}$ inversion time $[\mathrm{s}]$ & - & 53 & 21 & - & 163 & 33 & - & 342 & 54 \\
\hline K sparsification time [s] & - & 66 & 1.3 & - & 131 & 1.7 & - & 216 & 2.1 \\
\hline$\tau_{t}$ for $\mathbf{K}$ sparsification & - & $15 \cdot 10^{-5}$ & - & - & $112 \cdot 10^{-6}$ & - & - & $103 \cdot 10^{-6}$ & - \\
\hline Sparsity of K [\%] & - & 98.36 & 98.35 & - & 98.39 & 98.39 & - & 98.48 & 98.4 \\
\hline Memory occupancy $\mathbf{K}$ (or $\mathbf{L}_{p}$ for MNA) [MB] & 1046 & 17.5 & 17.6 & 2131 & 34.7 & 34.7 & 3520 & 55 & 57 \\
\hline $\mathbf{P}$ filling time $[\mathrm{s}]$ & 60 & 60 & 33 & 95 & 95 & 49 & 141 & 141 & 65 \\
\hline $\mathbf{P}$ inversion time $[\mathrm{s}]$ & 11 & 11 & 3 & 28 & 28 & 4 & 49 & 49 & 5 \\
\hline C sparsification time $[\mathrm{s}]$ & - & 11 & 0.5 & - & 18 & 0.6 & - & 27 & 0.7 \\
\hline$\tau_{t}$ for $\mathbf{C}$ sparsification & - & $13 \cdot 10^{-5}$ & - & - & $93 \cdot 10^{-6}$ & - & - & $76 \cdot 10^{-6}$ & - \\
\hline Sparsity of $\mathbf{C}[\%]$ & - & 98.36 & 98.36 & - & 98.42 & 98.42 & - & 98.48 & 98.41 \\
\hline Memory occupancy $\mathbf{C}[\mathrm{MB}]$ & 142 & 4.77 & 4.77 & 243 & 7.7 & 7.7 & 363 & 12 & 12 \\
\hline $\mathbf{F}_{k}$ (or $\mathbf{F}$ for MNA) LU decomposition time [s] & 100 & 24 & 1 & 290 & 65 & 1.8 & 785 & 136 & 3.43 \\
\hline Solution time* $[\mathrm{s}]$ & 5849 & 369 & 24 & 11046 & 810 & 44 & 19018 & 1332 & 72 \\
\hline Overall time [s] & 10478 & 5052 & 1898 & 20188 & 10039 & 3678 & 34506 & 16756 & 5959 \\
\hline
\end{tabular}

* total time for all the time samples.
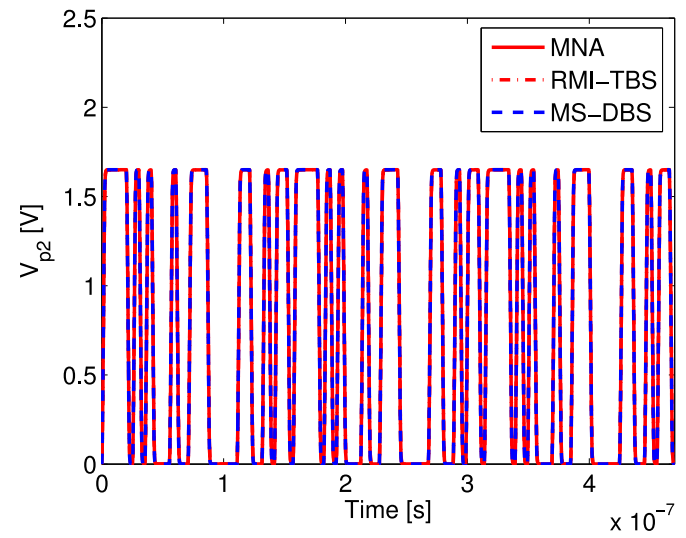

Fig. 8. Port 2 voltage for the irregular power bus example.

the sparsification performed by the MS-DBS algorithm is based only on the geometrical distance between the basis functions, and as a consequence, the $\mathbf{F}_{k}$ matrix [defined in (7)] is characterized by an higher locality. The proposed approach allows us to avoid the explicit scan of the $\mathbf{K}$ and $\mathbf{C}$ matrices, thus leading to a significant reduction of the sparsification time even for moderate-size problems. Furthermore, no effort is needed to identify the sparsification threshold. Finally, Figs. 6 and 8 show that the small approximation introduced by the compression of $\mathbf{L}_{p}$ and $\mathbf{P}$ matrices, and the sparsification of $\mathbf{K}$ and $\mathbf{C}$ matrices does not affect significantly the accuracy of the time-domain solution.

\section{CONCLUSION}

In this paper, a fast sparse reluctance- and capacitance-based solver for the PEEC method has been presented. For strongly interconnected structures and uniform meshes, the low-rank nature of matrices $\mathbf{L}_{p}$ and $\mathbf{P}$ is exploited to sparsify the reluctance matrix $\mathbf{K}$ and the capacitance matrix $\mathbf{C}$. The sparsified global PEEC system is efficiently solved by using the sparse 
multifrontal LU factorization. Furthermore, the multiscale block decomposition algorithm is used to accelerate the computation of the $\mathbf{K}$ and $\mathbf{C}$ matrices. In this case also, the low-rank nature of matrices $\mathbf{L}_{p}$ and $\mathbf{P}$ is exploited by the ACA algorithm, leading to both a satisfactory compression of the matrices themselves and a significant acceleration of their computation. Numerical experiments have been presented to demonstrate the efficiency and the accuracy of the proposed approach.

\section{REFERENCES}

[1] A. E. Ruehli, "Equivalent circuit models for three dimensional multiconductor systems," IEEE Trans. Microw. Theory Tech., vol. MTT-22, no. 3, pp. 216-221, Mar. 1974.

[2] W. C. Chew, J.-M. Jin, E. Michielssen, and J. Song, Fast and Efficient Algorithms in Computational Electromagnetics. Norwood, MA, USA: Artech House, 2001.

[3] V. Rokhlin, "Rapid solution of integral equations of classical potential theory," J. Comput. Phys., vol. 60, pp. 187-207, Sep. 1985.

[4] L. Greengard and V. Rokhlin, The Rapid Evaluation of Potential Fields in Particle Systems in Three Dimension. (Lecture Notes in Mathematics). Berlin, Germany: Springer, 1987.

[5] R. Coifman, V. Rokhlin, and S. Wandzura, "The fast multipole method: A pedestrian description," IEEE Antennas Propag. Mag., vol. 35, no. 3, pp. 7-12, Jun. 1993.

[6] C. C. Lu and W. C. Chew, "A multilevel algorithm for solving a boundary integral equation of wave scattering," Microw. Opt. Technol. Lett., vol. 7, no. 10, pp. 456-470, Jul. 1994.

[7] J. M. Song and W. C. Chew, "Multilevel fast-multipole algorithm for solving combined field integral equations of electromagnetic scattering," $\mathrm{Mi}$ crow. Opt. Technol. Lett., vol. 10, pp. 14-19, 1995.

[8] J. M. Song, L. Cai Cheng, and W. C. Chew, "Multilevel fast-multipole algorithm for electromagnetic scattering by large complex objects," IEEE Trans. Antenn. Propag., vol. 45, no. 10, pp. 1488-1493, Oct. 1997.

[9] S. Kapur and D. E. Long, "IES3: A fast integral equation solver for efficient 3-dimensional extraction," in Proc. Int. Conf. Comput. Aided Design, San Jose, CA, USA, Nov. 1997, pp. 448-455.

[10] K. Zhao and J.-F. Lee, "A single-level dual rank IE-QR algorithm to model large microstrips antenna arrays," IEEE Trans. Antennas Propag., vol. 52, no. 8, pp. 2580-2585, Oct. 2004.

[11] D. Gope and V. Jandhyala, "Efficient solution of EFIE via low-rank compression of multilevel predetermined interactions," IEEE Trans. Antennas Propag., vol. 53, no. 10, pp. 3324-3333, Oct. 2005.

[12] M. Bebendorf, "Approximation of boundary element matrices," Numer. Math., vol. 86, pp. 565-589, Jun. 2000.

[13] K. Zhao, V. Vouvakis, and J.-F. Lee, "The adaptive cross approximation algorithm for accelerated method of moment computations of EMC problems," IEEE Trans. Electromagn. Compat., vol. 47, no. 4, pp. 763-773, Nov. 2005.

[14] G. Antonini, S. Cristina, and A. Orlandi, "PEEC modeling of lightning protection system and coupling to coaxial cables," IEEE Trans. Electromagn. Compat., vol. 40, no. 4, pp. 481-491, Nov. 1998.

[15] A. E. Ruehli and A. C. Cangellaris, "Progress in the methodologies for the electrical modeling of interconnect and electronic packages," Proc. IEEE, vol. 89, no. 5, pp. 740-771, May 2001.

[16] P. Restle, A. E. Ruehli, S. Walker, and S. G. Papadopoulos, "Full-wave PEEC time-domain method for the modeling of on-chip interconnects," IEEE Trans. Comput.-Aided Design, vol. 20, no. 7, pp. 877-886, Jul. 2001.

[17] G. Antonini and A. E. Ruehli, "Fast multipole and multi-function PEEC methods," IEEE Trans. Mobile Comput., vol. 2, no. 4, pp. 288-298, Oct.Dec. 2003.

[18] G. Antonini, "Fast multipole method for time domain PEEC analysis," IEEE Trans. Mobile Comput., vol. 2, no. 4, pp. 275-287, Oct./Dec. 2003.

[19] D. Gope, A. E. Ruehli, and V. Jandhyala, "Speeding up PEEC partial inductance computation using a QR-based algorithm," IEEE Trans. Very Large Scale Integr. Syst., vol. 15, no. 1, pp. 60-68, Jan. 2007.

[20] A. Ruehli, D. Gope, and V Jandhyala, "Block-partitioned Gauss-Seidel PEEC solver accelerated by QR-based coupling matrix compression techniques," in Proc. IEEE 13th Top. Meet. Electr. Perform. Electron. Packag., Oct. 2004, pp. 325-328.
[21] H. Ji, A. Devgan, and W. Dai, "KSim: A stable and efficient RKC simulator for capturing on-chip inductance effect," in Proc. Asia South Pacif. Design Automat. Conf., Jan. 2001, pp. 379-384.

[22] Y. Du and W. Dai, "Partial reluctance based circuit simulation is efficient and stable," in Proc. Asia South Pacific Design Automat. Conf., vol. 1, 2005, pp. 483-488.

[23] J. Vlach, K. Singhal, Computer Methods for Circuit Simulation. New York, NY, USA: Van Nostrand Reinhold, 1983.

[24] S. Thamm, S. V. Kochetov, G. Wollenberg, and M. Leone, "PEEC modeling for EMC-relevant simulations of power electronics," in Proc. 17th Int. Conf. Radioelektronika, Apr. 2007, pp. 1-5.

[25] S. Thamm, S. V. Kochetov, G. Wollenberg, and M. Leone, "Alternative PEEC modeling with partial reluctances and capacitances for power electronics applications," in Proc. 7th Int. Symp. Electromagn. Compat. Electromagn. Ecol., Jun. 2007, pp. 56-59.

[26] D. Daroui and J. Ekman, "Efficient PEEC-based simulations using reluctance method for power electronic applications," Appl. Comput. Electromagn. Soc. J., vol. 27, no. 10, pp. 830-841, 2012.

[27] A. Devgan, H. Ji, and W. Dai, "How to efficiently capture on-chip inductance effects: Introducing a new circuit element $K$," in Proc. Int. Conf. Comput. Aided Design, 2000, pp. 150-155.

[28] A. Heldring, J. Rius, J. Tamayo, J. Parron, and E. Ubeda, "Multiscale compressed block decomposition for fast direct solution of method of moments linear system," IEEE Trans. Antennas Propag., vol. 59, no. 2, pp. 526-536, Feb. 2011

[29] G. Antonini and D. Romano, "Efficient frequency-domain analysis of PEEC circuits through multiscale compressed decomposition," IEEE Trans. Electromagn. Compat., vol. 56, no. 2, pp. 454-465, Apr. 2014.

[30] C. D. Meyer, Matrix Analysis and Applied Linear Algebra, Philadelphia, PA, USA: SIAM, 2001.

[31] T. A. Davis and I. S. Duff, "An unsymmetric-pattern multifrontal method for sparse LU factorization," SIAM J. Matrix Anal. Appl., vol. 18, no. 1, pp. 140-158, Jan. 1997.

[32] I. S. Duff and J. Koster, "The design and use of algorithms for permuting large entries to the diagonal of sparse matrices," SIAM J. Matrix Anal. Appl., vol. 20, no. 4, pp. 889-901, 1999.

[33] I. S. Duff and J. Koster, "On algorithms for permuting large entries to the diagonal of a sparse matrix," SIAM J. Matrix Anal. Appl., vol. 22, no. 4, pp. 973-996, 2001.

[34] E.-P. Li, E.-X. Liu, L.-W. Li, and M.-S. Leong, "A coupled efficient and systematic full-wave time-domain macromodeling and circuit simulation method for signal integrity analysis of high-speed interconnects," IEEE Trans. Adv. Packag., vol. 27, no. 1, pp. 213-223, Feb. 2004.

[35] R. Wang and J.-M. Jin, "A flexible time-stepping scheme for hybrid field-circuit simulation based on the extended time-domain finite element method," IEEE Trans. Adv. Packag., vol. 33, no. 4, pp. 769-776, Nov. 2010.

[36] G. Antonini and D. Romano, "Acceleration of time-domain nodalanalysis of partial element equivalent circuits through multiscale compressed decomposition," IEEE Trans. Electromagn. Compat., 2014, doi:10.1109/TEMC.2013.2294964

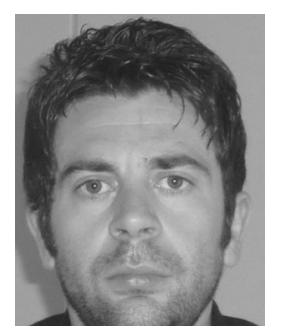

Daniele Romano was born in Campobasso, Italy, in 1984. He received the Laurea degree in computer science and automation engineering from the Università degli Studi dell'Aquila, L'Aquila, Italy, in 2012.

Since July 2012, he has been with the UAq EMC Laboratory, Università degli Studi dell'Aquila, focusing on algorithm engineering and speed-up techniques applied to large electromagnetic compatibility problems.

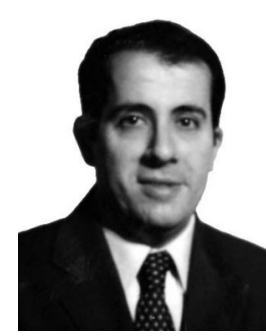

Giulio Antonini (M94-SM05) received the Laurea degree (summa cum laude) in electrical engineering from the Università degli Studi dell' Aquila, L'Aquila, Italy, in 1994 and the Ph.D. degree in electrical engineering from University of Rome "Sapienza," Roma, Italy, in 1998 .

Since 1998, he has been with the UAq EMC Laboratory, Università degli Studi dell'Aquila, where he is currently a Full Professor. 


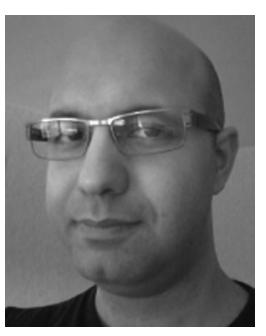

Danesh Daroui (M'12) received the M.Sc. degree from Gothenburg University, Gäteborg, Sweden, in 2008, and the Ph.D. degree in industrial electronics from Luleå University of Technology, Luleå, Sweden, in 2013.

$\mathrm{He}$ is currently a Researcher at the SP Technical Research Institute of Sweden, Borås, Sweden. His research interests include computational electromagnetics, partial element equivalent circuit modeling, and numerical analysis.

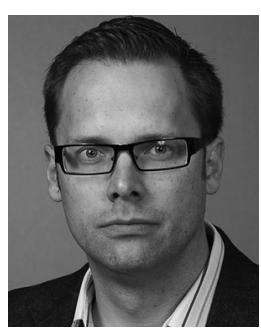

Jonas Ekman was born in Boden, Sweden, in 1972 He received the Ph.D. degree in electrical engineering from the Luleå University of Technology, Luleå, Sweden, in 2003.

From 2003 to 2007, hewas a Researcher at the Luleå University of Technology. in 2005 and 2006, he did his postdoctoral research in full-wave, time domain, partial element equivalent circuit (PEEC) modeling at the EMC Laboratory, University of L'Aquila, L'Aquila, Italy. In 2008, he was appointed an Associate Professor at the Luleå University of Technology. His research interests include computational electromagnetics, in particular, the use of the PEEC method for realistic electromagnetic modeling. 